\title{
Algunas características psicológicas de alumnos con problemas académicos
}

\author{
Edwin Salas \& Enrique Vera
}

Universidad de Lima Lima, Perú

La investigación tiene como finalidad identificar algunos de los factores que determinan los problemas académicos de estudiantes universitarios. Se estudiaron los informes psicológicos de 357 alumnos que reportaron esta situación entre abril de 1990 y agosto de 1996. Para identificar dichos factores se eligieron tres áreas de estudio: la inteligencia, evaluada con la Escala de Inteligencia de Wechsler para Adultos (WAIS); la personalidad, con el Inventario de Temperamento de Guilford-Zimmerman y el Inventario Multifásico de Personalidad de Minnesota (MMPI); y, los hábitos de estudio con la Encuesta de Habilidades de Estudio de Brown.

problemas académicos / bajo rendimiento / capacidad intelectual / habilidades para el estudio

\section{Some psychological characteristics of students undergoing academic problems}

Three hundred fifty-seven university records of students reported as undergoing academic problems were studied between Abril 1990 and August 1996. Research was aimed at determining the contributing factors to the problem and also at designing academic support programs and psychopedagogical support actions, and others on a preventive basis. Three study areas were selected: intelligence, as assessed through the WAIS test; personality as assessed through both the GuilfordZimmerman Temperament and the MMPI Inventories; and study habit, through Brown's Study Abilities Survey.

academic problems / low achievement / intellectual capacity / study abilities 


\section{INTRODUCCIÓN}

Cuando se estudia el rendimiento académico, se debe precisar que ésta es la expresión del aprendizaje escolar o universitario, que se mide a través de indicadores cuantitativos o notas $(0$ a 20 en nuestro sistema), o de algunas cualidades (aprobado A, B y C; desaprobado D y E). El rendimiento académico es una variable multicausada, en ella influyen muchos factores relacionados con los métodos de enseñanza y de aprendizaje, con la naturaleza de los cursos, con variables del docente, del propio estudiante y de otras de carácter medioambiental y de situaciones socioeconómicas. En la presente investigación se estudian descriptivamente tres factores que intervienen en el comportamiento académico de un grupo de estudiantes que solicitó consulta por presentar problemas académicos: inteligencia, personalidad y hábitos de estudio.

En relación con la inteligencia y el rendimiento académico existen varias líneas de investigación; así, por ejemplo, Pollit (1974, 1982, 1996); Majluf (1984, 1988, 1993); Alarcón (1986), entre otros, quienes vincularon el desarrollo intelectual y cognoscitivo en general con las condiciones de pobreza y desnutrición infantil. Bajo ese supuesto postularon que la inteligencia condiciona un tipo de rendimiento eficiente o deficiente en una actividad de tipo académico y cognoscitivo.

Estos trabajos sirvieron de base para el desarrollo de la investigación nacio- nal en esta área. Sin embargo, hay estudios que se relacionan mejor con el presente trabajo, tanto por el tipo de participantes como por el instrumento y los problemas que se abordan. Entre ellos están Casas y Cortés (1995), quienes con la Escala de Inteligencia de Wechsler para Adultos (WAIS) desarrollaron un perfil intelectual de jóvenes estudiantes de una universidad privada que presentaban desorientación vocacional. En su estudio concluyen que los estudiantes desorientados vocacionalmente tienen en promedio un perfil intelectual superior al normal. A su vez, Vera y Saénz (1997) utilizaron el mismo instrumento y estudiaron la capacidad intelectual en alumnos de bajo rendimiento en matemática, llegando a la conclusión de que estos jóvenes poseen una buena capacidad intelectual y que la inteligencia no se relaciona con los problemas de rendimiento que presentan.

Desde la perspectiva de la psicología cognitiva, Gonzales (1984) considera que los estudiantes desarrollan un fenómeno que se denomina "analfabetismo funcional". Luego Quesada y Gonzales (1985) estudiaron la comprensión lectora de estudiantes secundarios $\mathrm{y}$ universitarios de Lima. Demostraron la existencia de dicho fenómeno entre los alumnos que participaron en la investigación, encontrando que el problema se hace más agudo en la comprensión de textos numéricos, literarios, científicos, humanísticos y de carácter crítico, 
y que es menos intenso en las áreas de comprensión de textos informativos y documentarios. Por su parte, Pinzás (1997) analiza el problema de la lectura en nuestro sistema escolar y lo califica de grave y preocupante. Ella señala que la escuela no cumple con su función de enseñar a leer comprensivamente y esta realidad no sólo constituye un freno para el desarrollo académico, sino que también es una limitación para integrarse a un mundo en el que la información ocupa, cada vez más, un papel de mayor importancia. Del mismo modo, Gonzales (1998) trabajó la comprensión lectora en estudiantes universitarios iniciales de una institución privada y la contrastó con otro grupo similar de una universidad estatal. La investigación le permitió constatar que, en general, los estudiantes poseen menor dificultad comprensiva en textos documentarios, de información y numéricos, mientras que se incrementa cuando se trata de textos humanísticos, literarios y científicos. Al respecto, Tapia (1999) reporta un trabajo que realizó con un grupo de estudiantes secundarios del cuarto y quinto grados de un colegio estatal; dividió la muestra entre malos y buenos lectores y aplicó un programa remedial de recuperación pedagógica a un grupo experimental. Los resultados indicaron que si se enseña a los niños las técnicas de lectura, mejorarán su capacidad de comprensión; asimismo, que los estudiantes "malos lectores" sometidos a la experiencia, logran superar sus problemas iniciales.
Otro de los factores bastante estudiados en relación con los problemas del rendimiento académico es la personalidad. Así lo consideran algunos investigadores, como, por ejemplo, Bravo de Rueda (1988), quien aplicó el 16PF para comparar dos grupos de estudiantes de universidades limeñas y encontró que existe una especie de personalidad básica entre los sujetos de bajo rendimiento. Fernández (1990), comparó con el mismo instrumento los perfiles de personalidad de dos grupos de jóvenes estudiantes universitarios del primer ciclo que fueron agrupados sobre la base de su rendimiento (alto y bajo rendimiento) y constató que, en general, los estudiantes presentan ciertas actitudes negligentes y son poco constantes. Igualmente, los alumnos de alto rendimiento son más intranquilos y tensos; analíticos, independientes y autosuficientes; mientras que los de bajo rendimiento son mas bien prácticos, insensibles y libres de ansiedad. Álvarez (1994) estudió los valores interpersonales en estudiantes que se inician en la universidad y los relacionó con el rendimiento académico. Salas (1994), en un trabajo similar, estudió los perfiles de personalidad de los alumnos universitarios a través del inventario de Guilford y Zimmerman, y, posteriormente, con el mismo instrumento, analizó en 1995 las características de personalidad de un grupo de alumnos con problemas vocacionales. En estos estudios se encuentra que la benevolencia y 
reflexión aparecen con puntuaciones relativamente bajas; la ascendencia, el liderazgo y la sociabilidad con tendencia a los puntajes altos y con cierta insensibilidad y dureza, especialmente en las mujeres.

Hay también quienes consideran que el rendimiento está asociado a un conjunto de variables de la personalidad más específicos, como la autoestima y el concepto de sí mismo. Es el caso de Entwitle (1988), quien apunta que un factor muy importante para que un estudiante rinda adecuadamente, es el concepto y la estima que éste tenga de sí mismo. Enríquez (1999) trabajó el autoconcepto y la ansiedad ante los exámenes como predictores del rendimiento, para lo cual utilizó el inventario de autoevaluación sobre exámenes (Idase) y lo aplicó a 500 estudiantes secundarios de Lima, encontrando correlaciones positivas entre autoconcepto y rendimiento y negativas entre ansiedad ante los exámenes y rendimiento académico.

En esta línea de trabajos sobre personalidad, se ha planteado la hipótesis que supone que, en el fondo de los problemas académicos, se esconden en realidad problemas de personalidad. Con esta idea de base encontramos algunos trabajos que se relacionan de alguna forma con el nuestro. Así, García (1983) y Macedo (1994) abordaron por separado, en trabajos independientes y con diferentes grupos de estudiantes, las relaciones entre la ansiedad y el rendimiento académico, y encontraron correlaciones entre ambos factores. Del mismo modo lo hizo Rengifo (1995), quien investigó la personalidad de estudiantes que presentaban desorientación vocacional, a través del MMPI.

Con respecto a los hábitos de estudio, se han realizado un conjunto importante de investigaciones. Así, Adriazola (1990) analizó los hábitos de estudio de alumnos de una universidad privada, y encontró que éstos no son los más adecuados para desarrollar exitosamente los estudios universitarios. Por su parte, Salas (1989 y 1992) aplicó el inventario de habilidades de estudio de Brown a alumnos iniciales, también de una universidad privada, hallando que tanto en la organización como en las técnicas para estudiar, así como en la motivación, obtienen resultados calificados como inferiores, siendo el problema mayor la organización para estudiar. Vera y Winder (1995) aplicaron el mismo instrumento en un grupo de estudiantes que buscaron ayuda psicológica por presentar problemas de tipo vocacional; las puntuaciones, al igual que en las investigaciones anteriores, están por debajo de las normas y son inadecuadas para el trabajo universitario.

Por otro lado, existe numerosa bibliografía que se ha publicado en el área de análisis y que nos permite plantear algunas soluciones para los problemas vinculados con los estudios universitarios que, por lo demás, no son sólo un 
problema nacional, sino que sobrepasan nuestras fronteras. Ahí están los trabajos de Chávez y Mouchard (1988), Hernández (1993), Aduna y Márquez (1996), Gagné (1987), entre otros, quienes han elaborado todo un conjunto de conocimientos y tecnologías para abordar estos problemas íntimamente relacionados con el rendimiento académico universitario.

A pesar de que existen muchos trabajos que asocian la inteligencia, la personalidad o los hábitos de estudio con el rendimiento académico, no se han encontrado antecedentes de estudios en los que se aborde, con el mismo grupo de sujetos, el estudio de las variables antes mencionadas, ni descriptivamente ni a través de correlaciones o de un análisis factorial. En ese sentido, los datos que proponemos pueden ser de utilidad para tener una comprensión más cabal de los hechos a los que este trabajo se refiere.

\section{MéTodo}

\section{Participantes}

Se seleccionaron 357 informes psicológicos de estudiantes que buscaron orientación académica entre abril de 1990 y agosto de 1996. Estos alumnos, de todas las facultades y niveles de estudios, asistieron voluntariamente al servicio psicopedagógico y, en la entrevista inicial, refirieron tener problemas académicos (bajo rendimiento, insatis- facción con su desempeño, repitencias de cursos, que, a su vez, se relacionan con problemas de atención, concentración, comprensión lectora, etc). Se seleccionaron para la evaluación sólo los expedientes que contaban con el informe psicológico terminado y completo.

El grupo quedó constituido por $43 \%$ de mujeres y $57 \%$ de varones, de los cuales $22 \%$ cursaban estudios en el Programa de Estudios Generales, y el resto pertenecía a las facultades de Ingeniería Industrial (24\%), Administración (14\%), Derecho (10\%), Ciencias de la Comunicación (8\%) Ingeniería de Sistemas (8\%), Economía (7\%), Contabilidad (5\%) Psicología e Ingeniería Metalúrgica (1\%). Asimismo, considerando las categorías de pago, los alumnos estaban registrados de la siguiente manera: E-29\%, D-23\%, F-14\%, C$13 \%$, G-10\%, B-7\%, A-3\% у H-1\%. Para someter los resultados de estos grupos al análisis estadístico, se consideró como condición que existan por lo menos 10 datos por grupo.

\section{Diseño de la investigación}

La investigación, por sus propósitos y por el tipo de análisis que se realiza, puede ser clasificada como no experimental y descriptiva (Kerlinger, 1988; Salkind, 1998), en la que se trata de analizar las causas de las dificultades académicas a partir de la selección de un grupo de participantes que muestran dichos problemas en las áreas de la vida mental y comportamental (inteli- 
gencia, personalidad y habilidades para el estudio). También se pretende establecer las características de estos alumnos en función de ciertas variables, como la facultad de procedencia, el sexo y la categoría de pago; finalmente, los resultados son comparados estadísticamente tomando en cuenta los diferentes valores de las variables mediadoras mencionadas, y luego se correlacionan todos los factores de las pruebas.

\section{Instrumentos}

Para evaluar las diferentes áreas, se utilizaron los siguientes instrumentos:

- Para identificar el perfil de la inteligencia se utilizó la escala WAIS (Wechsler, 1955).

- Para identificar las características de la personalidad con la finalidad de encontrar perfiles de una supuesta personalidad básica de los estudiantes con problemas académicos, se utilizó el inventario de GuilfordZimmerman (Higueras, 1972).

- Para determinar la presencia o ausencia de rasgos clínicos que subyacen en el fondo de la estructura de la personalidad de jóvenes con problemas de rendimiento académico, se utilizó el MMPI forma R, adaptada para América Latina por Núñez (1981).

- Para analizar los hábitos de estudio e identificar sus principales aspectos se utilizó la encuesta William Brown, traducida y adaptada por García (1965).

\section{Procedimientos estadísticos}

En función de los objetivos del estudio, para el tratamiento estadístico y el análisis de los datos, se utilizaron las siguientes herramientas estadísticas:

- La media aritmética, que permitió agrupar los resultados de las diferentes áreas y en función de las variables consideradas.

- La prueba "T" de Student, para comparar si existían diferencias estadísticamente significativas entre dos grupos de datos.

- La prueba del rango de Duncan, para observar si existían diferencias estadísticamente significativas entre datos que provenían de más de dos grupos.

- El coeficiente de correlación de Pearson, para observar si entre los diferentes factores de las pruebas consideradas existían o no correlaciones que podían considerarse relevantes.

- Y las tablas y los gráficos (perfiles) que permiten la presentación y lectura fácil de los datos provenientes de los diferentes instrumentos.

\section{RESUltados de LA ESCALA WAIS}

El análisis de las puntuaciones promedio alcanzadas por el grupo estudiado revela que los puntajes más altos del grupo se encuentran en los subtests de información y diseño de cubos (categoría normal brillante); en forma decreciente encontramos el siguiente orden 
de los subtests: información, diseño de cubos, comprensión, semejanzas, vocabulario, dígitos símbolos, aritmética y memoria de dígitos (categoría normal superior). Siguen a éstos los de completamiento de figuras, ordenamiento de figuras y composición de objetos (categoría normal). Esto nos permite afirmar con cierta seguridad que, en general, estos estudiantes si bien presentan problemas académicos (de rendimiento, atención, concentración y comprensión lectora, etc.), no son ocasionados por la intervención de variables de carácter intelectual. El gráfico $\mathrm{N}^{\circ} 1$ muestra el perfil construido con los puntajes equivalentes de los once factores que mide la escala WAIS.

\section{Perfil intelectual de acuerdo con el sexo}

Al comparar los aspectos intelectuales de acuerdo con el género de los alumnos, se encuentra que los varones poseen un mayor nivel de información y habilidad para formar conceptos con elementos no verbales y más capacidad para resolver problemas de naturaleza numérica. Asimismo, la capacidad para el manejo del vocabulario, para elaborar juicios, para recordar hechos recientes, para planificar y anticiparse a las situaciones y para organizar la experiencia, es igual en ambos grupos. Por otro lado, las mujeres superan a los varones en la coordinación óculo-motora a través del manejo de un doble sistema de símbolos. Cuando se comparan estadísticamente los datos de la inteligencia a través del sexo, se encuentra que existe diferencia estadísticamente significativa en el test de completamiento de figuras, que indica que los varones poseen una mayor habilidad visual que las mujeres para observar detalles.

\section{Gráfico $\mathbf{N}^{\circ} 1$}

Representaciones de los puntajes equivalentes en el WAIS

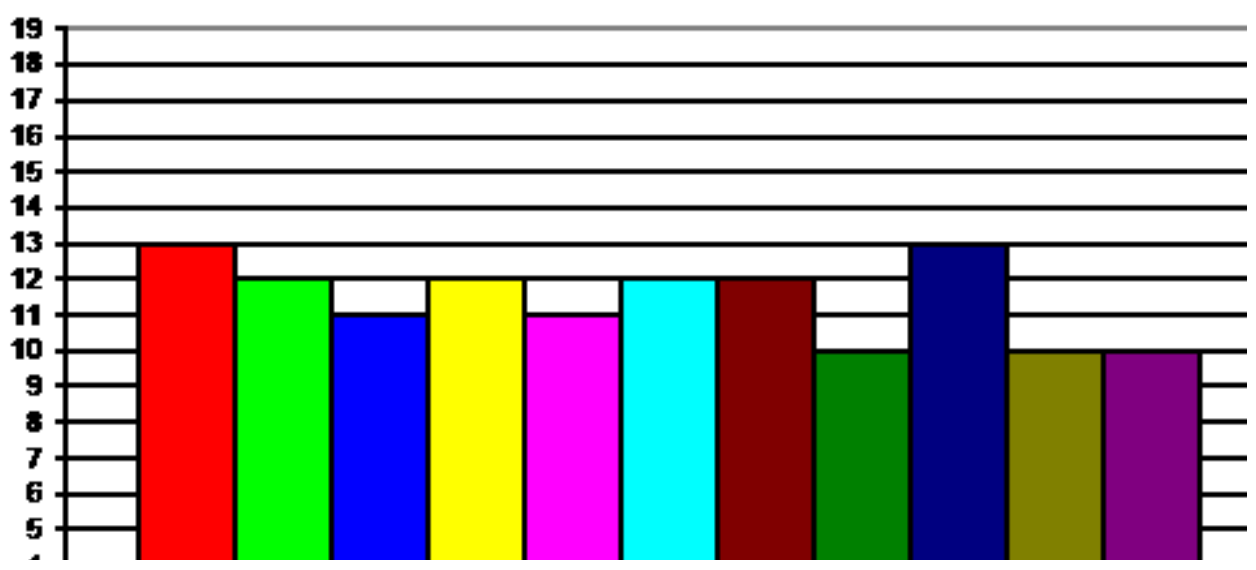




\section{Perfil intelectual de acuerdo con la facultad de procedencia}

Los niveles de información, cultura general e interés por el mundo circundante se ubican en un nivel normal brillante entre los alumnos de las facultades de Ciencias de la Comunicación, Administración, Economía, Ingeniería Industrial y Derecho; en el nivel promedio se ubican los estudiantes de Estudios Generales, Contabilidad e Ingeniería de Sistemas. En la capacidad para elaborar juicios y estimar lo que es apropiado en una situación determinada el grupo de alumnos de la Facultad de Derecho alcanza un nivel superior, seguido por los de Economía e Ingeniería de Sistemas que se encuentran en un nivel normal brillante; luego encontramos a los alumnos de las facultades de Ingeniería Industrial, Contabilidad y Administración; finalmente, en un nivel promedio, están los alumnos de Estudios Generales y Ciencias de la Comunicación.

La habilidad para resolver problemas aritméticos se ubica en un nivel normal brillante en los grupos de estudiantes de las facultades de Ingeniería de Sistemas y Contabilidad, seguidos por los de Derecho, Ingeniería Industrial y Ciencias de la Comunicación; en el nivel promedio se ubican los alumnos de Estudios Generales. En la capacidad para formar conceptos verbales destacan los alumnos de Ciencias de la Comunicación, Ingeniería de Sistemas, Derecho, Ingeniería Industrial, Admi- nistración y Estudios Generales, que se ubican en la categoría normal brillante.

La memoria reciente es superior entre los alumnos de las facultades de Economía, Ingeniería de Sistemas, Contabilidad e Ingeniería Industrial; en un nivel promedio se encuentran los de Ciencias de la Comunicación, Derecho, Administración y Estudios Generales. El vocabulario es normal brillante en los grupos de alumnos de Administración, Economía, Ciencias de la Comunicación, Ingeniería Industrial, Ingeniería de Sistemas, Contabilidad y Derecho; en el nivel promedio están los de Estudios Generales.

La coordinación óculo-motora es normal brillante en alumnos de las facultades de Ingeniería Industrial, Ingeniería de Sistemas, Economía, Contabilidad y Administración; es promedio para los de Estudios Generales, Ciencias de la Comunicación y Derecho. La capacidad para discriminar detalles es normal brillante para los jóvenes de las facultades de Ingeniería de Sistemas, Derecho, Ingeniería Industrial, Economía y Administración; en un nivel promedio encontramos a los de Ciencias de la Comunicación y Contabilidad, y en uno bajo a los de Estudios Generales.

La habilidad para formar conceptos con elementos no verbales es superior en el grupo de alumnos de Ingeniería Industrial; en un nivel normal brillante se ubican los alumnos de las facultades de Ingeniería de Sistemas, Economía y Contabilidad. En nivel promedio se hallan los de Derecho, Administración y 
Estudios Generales. La habilidad para anticiparse y planear los hechos es normal brillante en los alumnos de Ingeniería de Sistemas, Administración y Contabilidad. Los otros grupos están en el nivel promedio, siendo el grupo de Estudios Generales el que alcanza la menor puntuación dentro de este nivel. Finalmente, en cuanto a composición de objetos, los alumnos de todas las facultades, a excepción de los de Ingeniería de Sistemas, se ubican en el nivel promedio. En general se puede observar que las diferencias entre los datos por facultades pueden ser explicadas por su orientación vocacional, mas no creemos que sean válidos para aclarar los problemas del rendimiento y de otros asociados a éste. En varias de las capacidades y habilidades estudiadas se encuentran diferencias significativas entre los alumnos de las diversas facultades, materia que puede ser de utilidad para el consejo vocacional-profesional, no así para nuestros propósitos.

\section{Perfil intelectual según la categoría de pago}

El grupo de alumnos de la categoría A no figura en el presente análisis porque resultó ser más pequeño que la cifra considerada pertinente para enfocarlo estadísticamente (mínimo 10 datos). El nivel de información es normal brillante para los alumnos de las categorías $\mathrm{C}, \mathrm{E}, \mathrm{F}$ y $\mathrm{G}$ y promedio alto para las categorías de pago B y D. La capacidad de juicio, la comprensión y mo- vilización automática de los hechos es normal brillante para quienes pertenecen a las categorías $\mathrm{C}$ y $\mathrm{G}$; en un nivel promedio se ubican los grupos clasificados en B, D, F y E. En cuanto a la habilidad para resolver problemas aritméticos el grupo de la categoría $\mathrm{G}$ alcanza la puntuación más alta dentro de la categoría normal brillante, el resto de las categorías se ubican en un nivel normal promedio. En la habilidad para formar conceptos verbales todas las categorías comparten el nivel normal brillante, destacando la categoría C. La memoria de hechos recientes es superior en la categoría $\mathrm{E}$, mientras que las categorías $\mathrm{B}, \mathrm{C}$ y D se ubican en el nivel normal brillante.

En cuanto al conocimiento del significado de las palabras, los alumnos de todas las categorías comparten el nivel normal brillante, destacando el grupo de la categoría B. La coordinación óculo-motora y la habilidad para aprender un doble sistema de símbolos es normal brillante para todos. La habilidad para discriminar aspectos esenciales de la experiencia se encuentra en todos los alumnos de las diversas categorías en un nivel promedio, destacando la categoría B. Con referencia a la habilidad para formar conceptos con elementos no verbales haciendo uso de la capacidad de análisis y síntesis son los alumnos de las categorías $\mathrm{B}, \mathrm{C}$ y G los que ocupan los niveles más altos -superior-, seguidos por los otros grupos que obtienen un nivel normal brillante. 
La habilidad de los alumnos para planear y anticiparse a los hechos se ubica en un nivel promedio, ligeramente más alto para los alumnos de las categorías B y C. La capacidad para organizar experiencias, para ir de las partes hacia el todo, están en un nivel promedio entre todos los alumnos independientemente de la categoría de pago a la que pertenezcan.

Al hacer las comparaciones estadísticas vemos que se encuentran diferencias estadísticamente significativas en el test de aritmética, entre las categorías de pago $\mathrm{C}$ y $\mathrm{G}$, al igual que en semejanzas entre los alumnos de la escala B, quienes difieren de los de C. En la escala ejecutiva el análisis nos permite establecer que en dígitos-símbolo la escala de pago $G$ guarda una diferencia estadísticamente significativa con las categorías E, F, D, B y C. La escala E también tiene diferencias de este tipo con la categoría C. En completamiento de figuras la escala $\mathrm{F}$ tiene diferencias estadísticamente significativas con las categorías B y E. En composición de objetos, la escala de pago E guarda diferencias de la misma índole con las categorías D y C.

\section{Análisis de los Resultados del INVENTARIO GUILFORD ZIMMERMAN}

La tabla $\mathrm{N}^{\circ} 1$ contiene los datos generales hallados a través del inventario de Guilford-Zimmerman. Se puede apreciar que las puntuaciones medias convertidas a eneatipos alcanzan el calificativo medio (eneatipos 4, 5 y 6); sólo el factor dureza, que corresponde al sexo femenino, alcanza una calificación con eneatipo alto (7); acerca de este dato, Higueras (1972), quien adaptó la prueba en nuestro medio, propuso que este factor se tome como dureza y no como masculinidad, como podría darse

Tabla $\mathbf{N}^{\circ} 1$

Resultados generales del inventario Guilford-Zimmerman

\begin{tabular}{lccc}
\hline Factores & Media & Eneatipo & Calificación \\
\hline Actividad general & 16.41 & 5 & Medio \\
Refrenamiento & 16.13 & 5 & Medio \\
Ascendencia & 16.58 & 6 & Medio \\
Sociabilidad & 18.29 & 6 & Medio \\
Emocionabilidad & 13.46 & 5 & Medio \\
Objetividad & 14.48 & 4 & Medio \\
Benevolencia & 9.73 & 4 & Medio \\
Reflexión & 17.71 & 4 & Medio \\
Relaciones personales & 11.67 & 6 & Medio \\
Dureza Masculino & 16.8 & 5 & Medio \\
Dureza Femenino & 13.95 & 7 & Alto \\
\hline
\end{tabular}


a partir de la traducción de la prueba y de los manuales, con el fin de evitar las connotaciones e interpretaciones erróneas que el término masculinidad podría poseer en nuestra cultura.

En cuanto a la variable sexo, tanto las puntuaciones de los varones como las de las mujeres caen, en general, dentro del calificativo medio a excepción del factor dureza, en el cual las mujeres obtienen una calificación alta. Dentro de las puntuaciones medias, las mujeres alcanzan eneatipos más altos que los varones en los factores ascendencia, sociabilidad y relaciones personales. Las comparaciones estadísticas realizadas a través del " $\mathrm{T}$ " de Student no permiten encontrar diferencias estadísticamente significativas en los diversos factores de la prueba cuando se comparan los perfiles de los varones con los que corresponden a las mujeres.

\section{Resultados según la facultad de procedencia}

Los resultados obtenidos por este grupo de estudiantes presentan, en general, un perfil dentro de las calificaciones promedio (eneatipos 4,5 y 6 ) y reflejan perfiles ajustados de personalidad. Los alumnos de la Facultad de Administración presentan los puntajes más altos en el factor actividad general; en dureza se hallaron diferencias significativas entre los grupos de las facultades de Ingeniería Industrial y Derecho, siendo el primero el de más alto puntaje. Este dato puede ser indicativo del tipo de actividad que desarrollan ambos grupos de estudiantes.

\section{Resultados de acuerdo con la categoría de pago}

Si bien las puntuaciones de los estudiantes clasificados por categorías de pago caen en una zona del nivel medio, los alumnos de la categoría $\mathrm{C}$ obtiene un eneatipo más alto que los grupos de la categoría $\mathrm{D}$ y E en los factores actividad general y ascendencia; asimismo, el factor benevolencia resulta ser mayor entre estudiantes de la categoría D. No se encuentran diferencias significativas entre los resultados del Guilford-Zimmerman por categorías.

\section{ANÁlisis de los Resultados deL MMPI}

\section{Análisis de los resultados según el sexo}

En el gráfico $\mathrm{N}^{\circ} 2$ se encuentra que para el sexo masculino las escalas de depresión, psicastenia y esquizofrenia alcanzan en el perfil puntuaciones por encima de $\mathrm{T}$ (zona de patología). La hipomanía y la desviación psicopática se ubican exactamente en la zona limítro$\mathrm{fe}$, al igual que en la escala de validez $\mathrm{F}$.

En cuanto a los perfiles del sexo femenino, se caracteriza por tener puntuaciones que no llegan a sobrepasar el puntaje $T$, sin embargo, resultan altos en las escalas de depresión, desviación 
psicopática, esquizofrenia e hipomanía. En las escalas de paranoia y sociabilidad, las puntuaciones $\mathrm{T}$ de varones y mujeres son iguales, mientras que en las otras escalas los varones obtienen puntuaciones más elevadas en el perfil.

La depresión es indicador de disminución de la fuerza de voluntad, sentimientos de tristeza, desesperanza frente a la poca o nula posibilidad de manejar y controlar las situaciones, etc.; las manifestaciones ansiosas depresivas tienden a ser más evidentes en los alumnos varones que en las mujeres. En lo que se refiere a las escalas de sociabilidad encontramos que la escala de desviación psicopática es la más alta, tanto en varones como en mujeres; sin embargo, estas puntuaciones pueden ser explicadas tentativamente por la edad de los participantes investigados; en ambos grupos de alumnos las actitudes de rebeldía adolescente-juve- nil y los cambios ocasionados en los valores de estos grupos pueden estar provocando estos sesgos en los datos, que se materializan en términos de conducta a través de expresiones de disconformidad abierta ante diferentes situaciones, valores, creencias; en la crítica dura y a veces injustificada y en el cuestionamiento de las normas de conducta de la familia, de las instituciones, de la sociedad, etc. La escala de hipomanía advierte que los varones presentan mayores puntuaciones, lo que indica la presencia de una actividad desorganizada y sin orientación clara en la solución de sus problemas académicos; los intereses masculinos-femeninos revelan para ambos sexos intereses propios de cada uno y expresiones de sensibilidad por lo artístico, estético y plástico, características que bien pueden ser consideradas como parte de la personalidad adolescente y juvenil.

Gráfico $N^{\circ} 2$

Representación de los resultados del MMPI por sexo

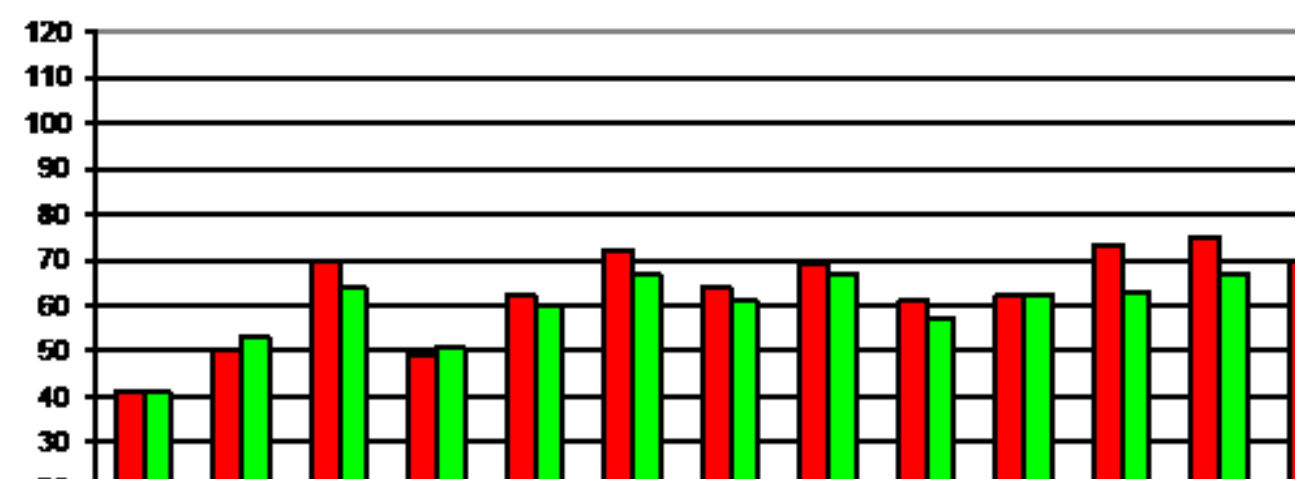


En lo que se refiere a la tétrada psicótica, en la escala de paranoia las puntuaciones $\mathrm{T}$ son iguales para los estudiantes en ambos sexos; sin embargo, en las otras tres escalas los varones obtienen puntajes más altos que las mujeres. Esto puede interpretarse como que la sensibilidad, la suspicacia, la alerta y los rasgos obsesivos compulsivos como indicadores de ansiedad son más elevados en los varones, ubicándose en la llamada zona de patología. Igualmente, las características relacionadas con la personalidad de tipo esquizoide se hacen más significativas en los varones, por lo que éstos tenderían a presentar más aislamiento, ensoñación y poco interés por establecer el contacto realista con los demás. Se debe destacar también que en el perfil del MMPI la puntuación media de los varones se ubica en la zona de patología, a diferencia de las mujeres, cuyas puntuaciones se encuentran en la zona de normalidad.

En una investigación realizada anteriormente con el MMPI por Rengifo (1995), quien trabajó con un grupo de estudiantes desorientados vocacionalmente, se encontraron resultados similares a los que se exponen en la presente investigación. En dicho caso la autora concluye que destacan los puntajes altos en las escalas de depresión, esquizofrenia, psicastenia, hipomanía y desviación psicopática; esto nos permite plantear que las características mencionadas en la actualidad pueden ser com- ponentes generacionales de estos jóvenes, independientemente de si tienen problemas de carácter vocacional u otros de carácter académico y de rendimiento. Cuando los resultados de nuestra investigación fueron sometidos a la prueba "T" de Student para realizar comparaciones entre los datos de las diversas escalas del MMPI en función de la variable sexo, no se encontraron diferencias estadísticamente significativas al valor del 0.05 .

\section{Resultados de acuerdo con la facultad de procedencia}

Para realizar este análisis se han calificado los resultados de todos los participantes (independientemente del sexo) y el perfil ha sido hallado con las normas de los varones; esto debe considerarse en la lectura de los datos que contiene este reporte. Los alumnos evaluados de todas las facultades, con excepción de los de Contabilidad, presentan una escala de depresión alta, en la que los alumnos de la Facultad de Economía alcanzan el puntaje más alto (T88), seguidos por el grupo de Ciencias de la Comunicación. La escala de esquizofrenia en todas las facultades se ubica en la zona de patología, con puntuaciones " $T$ " mayores a 80 . Con respecto a la escala de psicastenia, está por encima de $\mathrm{T}$ en todas las facultades, 10 que indicaría elevados índices de ansiedad en todos los alumnos.

En cuanto a la hipomanía, los alumnos del Programa de Estudios Genera- 
les y de las facultades de Contabilidad, Ingeniería Industrial y Derecho obtienen puntuaciones en la zona limítrofe (T70). Analizando las escalas de desviación psicopática se encuentra que los alumnos de Economía, Ciencias de la Comunicación e Ingeniería Industrial tienen puntuaciones por encima de T70. En la escala de hipocondriasis, Economía y Ciencias de la Comunicación obtienen puntuaciones que se ubican por encima de las normas.

Las comparaciones estadísticas permiten observar que existen diferencias significativas en la escala de hipocondriasis entre los alumnos de Ingeniería de Sistemas y Economía; igualmente entre los de Ciencias de la Comunicación, Contabilidad y Estudios Generales. En cuanto a la escala de histeria existen diferencias entre los jóvenes de Derecho y Ciencias de la Comunicación. En desviación psicopática se revela que hay diferencia entre los estudiantes de las facultades de Administración e Ingeniería Industrial; lo mismo ocurre en la escala de paranoia entre los jóvenes de Administración e Ingeniería de Sistemas. Finalmente, en la escala de esquizofrenia difieren los resultados de los alumnos de Ciencias de la Comunicación e Ingeniería Industrial.

\section{Resultados clasificados por categoría de pago}

Con relación a la depresión, los alumnos de las categorías de pago D, E y F tienen puntuaciones que se hallan en la zona de patología, siendo la más alta la de aquéllos ubicados en la categoría $\mathrm{D}$. En desviación psicopática obtienen puntuaciones por encima de $\mathrm{T}$ los alumnos de las categorías $\mathrm{D}$ (T75) y $\mathrm{F}$ (T72). En la escala de psicastenia, los jóvenes de todas las categorías de pago se ubican en la zona de patología; esto implicaría la presencia de conductas obsesivas compulsivas como forma de reducir la ansiedad. En hipomanía, son los estudiantes de la categoría $\mathrm{C}$ quienes se ubican en la zona de patología. En cuanto a sociabilidad todos los alumnos logran un equilibrio entre la introversión y la extroversión. En cuanto a las comparaciones estadísticas no se encuentran diferencias significativas entre los grupos.

\section{Resultados generales de la encuesta de habilidades del estudio de Brown}

Las calificaciones de las tres pruebas que componen este test nos muestran que los hábitos de estudio se encuentran en niveles bastante inferiores con relación a lo que se espera idealmente para un estudiante universitario. Las puntuaciones obtenidas por estos alumnos en las tres subpruebas, así como el puntaje total no llegan a superar con claridad el $50 \%$ del puntaje posible; la tabla $\mathrm{N}^{\circ} 2$ contiene los resultados obtenidos en la presente investigación. Se puede apreciar, asimismo, que aun cuando no sea significativa la diferencia entre las tres pruebas, el puntaje de 
la prueba de organización es el más bajo de los tres factores medidos.

Tabla $\mathbf{N}^{\circ} 2$

Resultados generales de la prueba de hábitos de estudio de Brown

\begin{tabular}{lcc}
\hline Prueba & Media & Calificación \\
\hline Organización & 9.5231 & Bajo \\
Técnicas & 10.3487 & Inferior \\
Motivación & 10.3641 & Bajo \\
Total & 30.299 & Inferior \\
\hline
\end{tabular}

Antes del presente reporte de investigación se han realizado otros estudios sobre los hábitos de estudio, utilizando el mismo instrumento y con estudiantes de la misma universidad. Salas (1989 y 1992) trabajó con estudiantes que iniciaban sus estudios universitarios y en estas dos investigaciones se muestra que las mediciones de la prueba de hábitos obtienen, en promedio, mejores puntajes que los del presente reporte, aun cuando cualitativamente y estadísticamente son poco importantes. Del mismo modo, Vera y Winder investigaron en 1995 los hábitos de estudio en alumnos que buscaron apoyo psicológico motivados por problemas de tipo vocacional. Los datos de las investigaciones mencionadas, en términos de sus resultados generales y de los que corresponden a cada una de las subpruebas de la encuesta sobre habilidades de estudio, se pueden encontrar en la tabla $\mathrm{N}^{\mathrm{o}} 3$.

Por otro lado, en cuanto al tipo de problemas que éstos desarrollan, hay muchas coincidencias con los estudios realizados; la diferencia radica, en todo caso, en el grado del problema. Los estudiantes que participaron en la presente investigación tienen problemas más pronunciados en el desarrollo de sus hábitos para estudiar, sobre todo en la organización y la motivación para el estudio. Vemos que en el subtest de organización los resultados de las tres anteriores investigaciones (véase tabla $\mathrm{N}^{\mathrm{O}}$ 3) tienen puntajes más altos que los encontrados en la presente ocasión, y que existe bastante cercanía entre los valores de las puntuaciones de esas tres primeras mediciones. La diferencia queda claramente establecida; es el tipo de participantes en cada uno de los estudios realizados. En la presente investi-

Tabla $N^{\circ} 3$

Datos obtenidos con la prueba de Brown en anteriores investigaciones con grupos similares

\begin{tabular}{lccclc}
\hline Medidas & Organización & Técnicas & Motivación & Total & N $^{\circ}$ alumnos \\
\hline Salas 1989 & 11.47 & 11.74 & 12.01 & 35.22 & 358 \\
Salas 1992 & 11.5 & 11.8 & 12.0 & 35.5 & 1818 \\
Vera 1995 & 11.1 & 11.9 & 11.04 & 34.04 & 50 \\
Inv. Actual & 9.52 & 10.35 & 10.36 & 30.30 & 198 \\
\hline
\end{tabular}


gación los sujetos manifestaban tener problemas académicos, mientras que en las anteriores fueron alumnos con problemas vocacionales o recién ingresados a la universidad.

En la subprueba técnicas para estudiar, los puntajes promedios obtenidos en las tres primeras investigaciones (véase tabla $\mathrm{N}^{\circ} 3$ ) son bastante similares, y los resultados de la presente investigación son los más bajos de todos los datos presentados. En la subprueba motivación para el estudio, podemos observar una tendencia similar a las pruebas anteriores, los datos son distintos entre los cuatro grupos de sujetos, pero hay que notar que los resultados de la presente investigación son los más bajos de todos ellos. Resulta interesante observar que los resultados de los estudios hechos con estudiantes ingresantes (Salas, 1989 y 1992) tienen en promedio mejores puntajes que los otros grupos que presentan problemas vocacionales (Vera y Winder, 1995) y los del presente estudio que, como reiteramos, tomó alumnos que manifestaron tener problemas académicos.

Revisando los puntajes totales encontramos que los resultados de las anteriores investigaciones son más altos que los del presente reporte. Creemos que los resultados nos permiten afirmar que estas diferencias pueden estar dadas por el tipo de alumnos que han conformado los tres estudios: en las investigaciones realizadas por Salas los sujetos que participaron fueron estudian- tes del primer nivel del Programa de Estudios Generales apenas después de haber comenzado sus estudios universitarios; en la investigación desarrollada por Vera y Winder, el otro grupo de estudiantes que participó en el estudio presentaba problemas de carácter vocacional; en tanto que los datos del presente trabajo provienen de estudiantes con problemas académicos. Entre éstos existen quienes poseen bajo rendimiento, que no están satisfechos con sus notas ni con su actuación como estudiantes; hay otros que presentan problemas al momento de dar exámenes, que no tienen buena comprensión y/o velocidad de lectura o no pueden manejar bien las técnicas para el estudio universitario, o no están motivados para estudiar en la universidad y prefieren una carrera técnica, etc.

\section{Resultados de la prueba de hábitos de estudio según el sexo}

En cuanto a los hábitos para estudiar no existen diferencias estadísticamente significativas entre los perfiles de los estudiantes varones y mujeres. Los resultados de las subpruebas son independientes del género de los estudiantes. Ambos grupos obtienen en organización, técnicas y motivación para el estudio, puntajes que se corresponden con una calificación en categoría inferior. 


\section{Resultados de acuerdo con la facultad de procedencia}

Los alumnos de la Facultad de Derecho tienen los mejores puntajes en las tres pruebas así como en el puntaje total. En la prueba de organización el puntaje mayor corresponde a los estudiantes de las facultades de Derecho y de Ingeniería de Sistemas y el más bajo a los de Economía. En técnicas para estudiar, nuevamente sobresalen los de Derecho y se repite el puntaje más bajo para los de Economía. En motivación, el mayor puntaje lo obtienen también los alumnos de la Facultad de Derecho y el más bajo corresponde a los del Programa de Estudios Generales. Igualmente, la observación de los puntajes totales nos permite afirmar que los alumnos de Derecho tienen los puntajes más sobresalientes y en Economía encontramos las calificaciones más bajas.

Como resultado de realizar comparaciones estadísticas entre las diferentes facultades, se encuentra que, en lo que se refiere a organización y técnicas para estudiar, no existen diferencias estadísticamente significativas entre los estudiantes de las diferentes facultades. En cuanto a la motivación sí se hallan diferencias entre Derecho y Estudios Generales, así como entre Derecho y Contabilidad.

\section{Resultados según la categoría de pago}

En organización, el mayor puntaje se da entre los alumnos de la categoría $\mathrm{C}$ y el más bajo es compartido por estudiantes de las categorías A y E. En la prueba referida a las técnicas para estudiar el puntaje que sobresale se da en la categoría $\mathrm{F}$ y el menor en la E. En cuanto a la motivación, la categoría $\mathrm{C}$ obtiene la mayor significación y la categoría $\mathrm{G}$ el menor nivel.

Al compararse estadísticamente los resultados en relación con las diferentes categorías de pago no se encuentran diferencias significativas.

\section{INTERPRETACIÓN Y DISCUSIÓN DE LOS RESULTADOS}

Debido a la gran cantidad de datos que se han recogido para este estudio, la labor de la interpretación requiere de mucho más espacio que con el que se cuenta, así como de mayores posibilidades para ensayar una y otra vez comentarios que puedan ser discutidos; por ello consideramos imprescindible declarar que, en realidad, esta parte del trabajo no puede ser sino un primer intento explicativo y que, a partir de éste, se pueden seguir construyendo hipótesis plausibles en la medida en que otros psicólogos, pedagogos e investigadores se interesen en el tema y profundicen sus observaciones sobre los datos que se presentan.

Un primer aspecto que queda claro y que puede explicar el bajo rendimiento y otros problemas académicos de estos estudiantes, es que desarrollan hábitos de estudio bastante inadecuados; como se señaló antes, otros grupos de estu- 
diantes que no presentan los problemas académicos manifestados por ellos, tienen mejores hábitos para estudiar que el grupo que hemos investigado. Dentro de esta problemática general, la incapacidad mayor se ubica en la organización, que, como bien sabemos, es la capacidad para administrar el tiempo, las tareas, las acciones previas al estudio y aquello que va a servir para mejorar las capacidades de atención, concentración y de estudio comprensivo. En relación con las técnicas para estudiar, que también se encuentran en niveles inadecuados, creemos que éstos y otros estudiantes universitarios conocen las estrategias y los procedimientos que deben seguir, el cómo hacer bien las cosas para estudiar y rendir bien. El problema de los jóvenes en general y en particular de los que participaron en este estudio, es que no hacen necesariamente lo que "deben" o "saben que deben de hacer". Existe, pues, un elemento disonante entre lo cognitivo, lo actitudinal y lo conductual, que también puede tener relación con una serie de fenómenos que limitan la solución de problemas como la fijación funcional y el acomodo mental. Uno de los problemas más graves al que se enfrenta el psicólogo o pedagogo que desea trabajar con este tipo de dificultades es, precisamente, cómo modificar el aspecto actitudinal de los alumnos; ellos no dan valor a la disciplina con que debe abordarse el estudio universitario, ni a los aspectos relacionados con la adminis- tración de los ambientes en los que desarrollan sus estudios, ni a su tiempo; tampoco a las técnicas para desarrollar adecuadamente trabajos académicos: fichas, esquemas, resúmenes, notas, etc.

Del mismo modo, el problema de la motivación es muy pronunciado en estos jóvenes; generalmente dependen de los incentivos, de motivos externos; están en la universidad pero no se encuentran comprometidos personalmente con lo que están realizando. Por eso no entienden el valor de algunos cursos con relación a la facultad y a la carrera profesional que eligieron, rechazan algunas clases de tareas (hacer fichas, resúmenes, leer sistemáticamente, etc.), manifiestan no tener aptitudes para aprobar un curso que no está relacionado de maneara directa con la profesión elegida, se retiran tempranamente de algún curso que les parece "difícil", etc. Los motivos más importantes que van a movilizar a los estudiantes en la universidad tienen que ser de naturaleza intrínseca, y éstos no han sido internalizados por nuestros jóvenes que presentan problemas académicos; es decir que los alumnos están controlados desde afuera y la universidad, por sus características y propio sistema, requiere que los alumnos estén motivados desde su interior.

En cuanto a los resultados de la personalidad a través del inventario de Guilford-Zimmerman, aparentemente los resultados caen, dentro de los pro- 
medios del tipo de población estudiada, en una zona "normal"; creemos, sin embargo, que los puntajes medios que acumulan en actividad general, objetividad, benevolencia, reflexión, no son muy adecuados para personas que desarrollan actividades intelectuales, racionales y reflexivas intensas. Si a esto le agregamos las tendencias medias altas en ascendencia, sociabilidad y dureza, podemos estar configurando un tipo de personalidad que actúa con cierto desparpajo y con tendencias de conducta más dependiente del exterior que del propio sujeto; incapaces de ordenarse y autogobernarse, de establecer una crítica objetiva de su propia acción o inacción; no por falta de capacidad intelectual, sino más bien por una evidente carencia de adecuación social y, por cierto, desorden en el accionar académico, producto de la experiencia anterior en torno a la dependencia-familia y escuela-, interpretación que tiene estrecha relación con los hallazgos en la prueba de hábitos para estudiar.

En cuanto a los resultados del MMPI, encontramos una fuerte dosis de ánimo depresivo en los varones, mientras que en las mujeres aparecen algunas tendencias a la somatización; la ansiedad aparece alta en los dos grupos de alumnos clasificados según el sexo; sin embargo, es más notoria en los varones. Estos primeros elementos del MMPI nos muestran a los jóvenes de este grupo como sujetos que tienen conciencia de su problema, lo que les causa ansie- dad con tendencia a la actividad, pero que, no obstante, no realizan las acciones correctas para mejorar su situación sobre la base de su propia voluntad. Esto produce en ellos sentimientos de tristeza, depresión y desesperanza que, al mismo tiempo, se constituyen en una especie de círculo vicioso perfecto: conciencia del problema, ansiedad, actividad desorganizada, frustración, desesperanza, depresión...

Algo que puede llamar poderosamente la atención son las puntuaciones altas en las escalas de sociabilidad, que nos informa que se trata de jóvenes con una desviación psicopática pronunciada. Sin embargo, creemos que se debe considerar, por un lado, la edad de estos estudiantes y lo que sabemos de la teoría del desarrollo del adolescente y su relación con las figuras de autoridad, y por otro, es necesario tener en cuenta cambios que se han operado en nuestra cultura, que no se han visto reflejados en una revisión de ítems de la prueba del MMPI. Si hacemos una lectura atenta de algunos de ellos, llegaremos a entender que la psicopatía no puede estar constituida ahora por las mismas conductas que hace veinte años.

En cuanto a otros factores que componen la tétrada psicótica, encontramos la configuración de un perfil que se corresponde con personas suspicaces, altamente sensibles, de rasgos esquizoides. Si tomamos en cuenta los hallazgos de las investigaciones previas con- 
sideradas en el marco teórico que vincula la personalidad con el rendimiento y los resultados de este estudio con la prueba de Guilford-Zimmerman, estaremos en capacidad de relacionarlos todos bajo la idea de un círculo vicioso: altas expectativas-bajos niveles de actividad y de rendimiento-inseguridadansiedad-suspicacia-bajo rendimiento e insatisfacción.

Por todo lo expuesto, podemos afirmar que sí existen algunos rasgos de personalidad que tipifican a los alumnos con problemas académicos y bajo rendimiento, pero no podemos asegurar que estos rasgos sean los que originan el bajo rendimiento o sean más bien resultantes precisamente del fracaso y de la frustración. Creemos por el momento, por lo menos, que es importante considerar que el rendimiento académico es un factor multicausado, idea que debe estar presente en toda acción del psicopedagogo que pretenda ayudar a niños y jóvenes que presentan estos problemas.

Finalmente, consideramos que, en términos generales, por lo menos en el caso del grupo con el que hemos trabajado, el bajo rendimiento no tiene que ver con la inteligencia de los estudiantes. Por el contrario, si nos atenemos al perfil intelectual que poseen, podrían ser considerados como potencialmente exitosos. Casi en todos los factores se ubican en los límites superiores de la normalidad o sobre éstos; en niveles normales y un tanto bajos para el pro- medio que el propio grupo obtiene, aparecen los resultados de los subtests que tienen que ver con la atención y la concentración, la capacidad de anticipación y de planeamiento, así como la capacidad de organización. Éstas son apreciaciones que podemos notar reiteradamente y relacionarlas con los otros resultados a los que se ha hecho referencia y que pueden estar afectados, precisamente, por las situaciones de contexto y por sensaciones y percepciones acerca de uno mismo, así como por dificultades para adecuarse rápidamente al ambiente universitario. A esto puede agregarse las situaciones emocionales mal manejadas por los participantes del estudio.

Pero estos alumnos no sólo se caracterizan por "aspectos negativos", ni el bajo rendimiento académico es un problema irremediable en ellos. Consideramos que existen muchos aspectos positivos que deben resaltarse y ser tomados en cuenta dentro de las acciones del departamento psicopedagógico $\mathrm{y}$ de la consejería universitaria. En primer lugar, la capacidad intelectual que poseen estos alumnos es alta y bastante adecuada para el desarrollo de actividades cognitivas en la universidad. Tienen una gran potencialidad para lograr grandes desempeños, potencialidad que en este momento no está siendo adecuadamente aprovechada debido a la presencia de numerosos factores que no les permiten a estos estudiantes superar las limitaciones en las que se en- 
cuentran. Además de la capacidad intelectual, la universidad exige una buena organización, dedicación, voluntad y motivación intrínseca, cualidades que se deben trabajar más sistemáticamente desde las unidades académicas de la universidad o desde dependencias de bienestar universitario. En este sentido, consideramos importante insistir en el desarrollo de programas que tengan como finalidad conseguir una adaptación más rápida del estudiante a las exigencias de la universidad. En segundo lugar, la ansiedad y los rasgos depresivos que en este momento constituyen un problema, son superables por el estudiante; la ansiedad aislada de los demás elementos conflictos, con la condición de que esté adecuadamente manejada, puede producir conductas de logro que ayuden a estos alumnos a mejorar su estima e imagen personal, a la vez que puede ser una condición para mejorar su capacidad de organización y gerencia de sus actividades y tiempo. En tercer lugar, estos estudiantes son conscientes de sus problemas, tienen ansiedad y sentido de culpa, pero requieren de la ayuda externa -por lo menos por el momento- para reorganizarse sobre la base de este apoyo. Por último, son personas muy jóvenes, que en su mayoría deben tener entre 18 y 20 años y, por lo tanto, se espera que ganen en madurez social, hecho que permitirá un desarrollo más adecuado a las circunstancias.

\section{Conclusiones}

- Los perfiles intelectuales de los estudiantes atendidos por presentar problemas académicos y de rendimiento, no permiten relacionar ambas variables, en todo caso se observa en el WAIS que los factores más bajos de los puntajes logrados se ubican en la categoría mental normal, que no los incapacita ni los hace deficientes, y tienen que ver con funciones específicas como la atención y concentración, así como con la organización, anticipación y planeamiento.

- Los perfiles de la personalidad a través del inventario de Guilford-Zimmerman permiten apreciar a un grupo de sujetos con puntuaciones absolutamente medias en todos los factores. si bien ello podría indicar la idea de "normalidad", en el análisis de las condiciones personales para el desarrollo de una profesión específica podría considerarse también como una limitación seria. Asimismo, a pesar de estar en la norma, los factores actividad general, benevolencia, reflexión y objetividad, no son los más adecuados para quienes desarrollan una actividad intelectual.

- Entre los perfiles del MMPI, se advierte la presencia de algunos componentes importantes: son bastante ansiosos, depresivos en el caso de los varones y con elevados índices de somatización en el caso de las mujeres. Los rasgos de una persona- 
lidad esquizoide y antisocial aparecen altos, lo cual es explicable por la edad de los sujetos y por los cambios culturales operados sobre nuestras ideas de la normalidad. Igualmente aparecen pronunciados algunos rasgos de la personalidad esquizoide. Del mismo modo, su actividad es desorganizada.

- Los resultados de la prueba de hábitos de estudio muestran a jóvenes con hábitos inadecuados para desarrollar estudios en el nivel en el que se encuentran. Los datos pueden explicar, en gran medida, por qué estos estudiantes se encuentran con problemas académicos.

- Integrando los resultados en una interpretación general, podría sostenerse que existe en el comportamiento de estos alumnos una necesidad de logro insatisfecha y a la vez una sensación de fracaso que los empuja a pedir ayuda, fracaso que los perturba emocional, social y cognitivamente y que los hace suspicaces, sensitivos, ansiosos. Esto, a su vez, los lleva a más preocupaciones en cuanto a sus capacidades y logros; una especie de círculo vicioso del cual no pueden salir por sí mismos.

\section{REFERENCIAS}

Adriazola, M. (1990). Los hábitos de estudio en los alumnos de la Universidad de Lima, según sexo y la facultad. Informe no publicado. Uni- versidad de Lima. Programa de Estudios Generales, Lima.

Aduna, A. \& Márquez, E. (1996). Curso de hábitos de estudio y autocontrol. México: Editorial Trillas.

Alarcón, R. (1986). Psicología, pobreza y subdesarrollo. Lima: Instituto Nacional de Investigación y Desarrollo Educativo.

Álvarez, D. (1994). Valores interpersonales y rendimiento académico en estudiantes ingresantes a la Universidad de Lima. Informe no publicado. Universidad de Lima, Lima.

Bravo de Rueda, J. (1988). Acción tutorial de estudiante a estudiante y rendimiento académico en una universidad particular de Lima Metropolitana. Tesis de licenciatura, Universidad Femenina del Sagrado Corazón, Lima.

Bravo de Rueda, M. del C. (1988). Factores de personalidad: 16 PF Catell y temores en estudiantes de psicología en dos universidades de Lima Metropolitana. Tesis de licenciatura Universidad Femenina del Sagrado Corazón, Lima.

Brown, W. (1975). Encuesta sobre habilidades de estudio. Traducción y adaptación de Eduardo García y Fernando G.C. Copia mimeografiada.

Brown, W. (1975). Guía de estudio efectivo. México: Editorial Trillas.

Casas, A. \& Cortés, M. (1995). Perfil intelectual en alumnos desorientados vocacionalmente, con elección vocacional congruente e incongruente. Informe no publicado. Arequipa, Primer Congreso Nacional de Psicología Educativa.

Chávez, J. \& Mouchard, T. (1989). 46 preguntas y respuestas sobre el estudio y el 
aprendizaje. Lima: Universidad de Lima, serie El Proceso de Aprendizaje 1.

Entwittle, N. (1988). La comprensión del aprendizaje en el aula. Buenos Aires: Editorial Paidós.

Enríquez Verau, Jorge Luis. (1999). El autoconcepto y la ansiedad ante los exámenes como predictores del rendimiento en estudiantes de secundaria de un colegio nacional de La Molina. Revista de Psicología III(1-2), 149-169. Lima: Universidad Inca Garcilaso de la Vega.

Fernández, M. (1990). Factores de personalidad y rendimiento académico. Un estudio comparativo. Cuadernos de Psicología I. Cuestiones de Psicología del Aprendizaje. Lima: Universidad de Lima, 127-200.

Gagne, R. (1987). Las condiciones del aprendizaje. 4a. edición. México: Editorial Interamericana.

García Zapatero, Giannina. (1983). Ansiedad debilitadora y rendimiento escolar. Revista de Psicología, I(1). Lima: Pontificia Universidad Católica del Perú.

Gonzales M., Raúl. (1984). El desarrollo psicolingüístico de las asociaciones verbales. Revista de Psicología, II(2), Lima: Pontificia Universidad Católica del Perú.

Gonzales M., Raúl. (1998). Comprensión lectora en estudiantes universitarios iniciales. Persona, 1, Universidad de Lima.

Gonzales M., Raúl \& Quesada, R. (1985). Algunos índices psicolingüísticos del lenguaje de los estudiantes de educación básica de Lima. Comportamiento, 3(4).

Hathaway, S.R. \& McKinley, J.C. (1981). Cuestionario de personalidad MMPI. Manual, forma $R$. Adaptación para América Latina por el doctor Rafael
Núñez. México: Editorial Manual Moderno.

Hernández, F. (1983). Métodos y técnicas de estudio en la universidad. Bogotá: McGraw-Hill.

Higueras, C. Leonardo. (1972). Manual del Inventario de Temperamento de Guilford Zimmerman. Lima: Edición Sios, Material y Publicaciones Psicométricas.

Kerlinger, Fred N. (1994). Investigación del comportamiento. 3a. edición. México: McGraw-Hill/Interamericana de México, S.A. de C.V.

Lazo Manrique, Jorge y Zanolo Martinuzzi, Bruno. (1991). Investigaciones Psicológicas en el Perú. 3a. edición. Lima: Editora Caribe. Biblioteca Andina de Psicología.

Majluf, Alegría. (1984). Rendimiento intelectual de niños, infantes y adolescentes. Revista de Psicología, II(2). Lima: Pontificia Universidad Católica del Perú.

Majluf, Alegría. (1988). Relación entre el estado de nutrición y la estimulación ambiental con el rendimiento cognitivo y escolar del niño de primer grado de la zona central media del Perú (costa, sierra y selva). Revista de Psicología, VI(6). Lima: Pontificia Universidad Católica del Perú.

Majluf, Alegría. (1993). Marginalidad, inteligencia y rendimiento académico. Lima: Editorial Brandon Enterprise S.R.Ltda.

Macedo Alfaro, Narda. (1994). Ansiedad frente a la matemática en un grupo de alumnos universitarios. Segundo Encuentro de Psicólogos y Orientadores en Educación Superior, Universidad de Lima. 
Pinzás G., Juana. (1997). Metacognición y lectura. Lima: Pontificia Universidad Católica del Perú.

Pollit, Ernesto. (1974). Desnutrición, pobreza e inteligencia. Lima: Retablo de Papel Ediciones, 1974.

Pollit, Ernesto. (1982). Desnutrición, inteligencia y política social. Lima: Librería Studium.

Pollit, Ernesto. (1996). Desayuno escolar y rendimiento; a propósito del programa de desayunos escolares de Foncodes en el Perú. Lima: Apoyo.

Rengifo, Glenda \& Moloche, María. (1995). Perfil de personalidad a través del MMPI en alumnos que solicitan orientación vocacional. Primer Congreso Nacional de Psicología Educativa (documento no publicado), Arequipa.

Salas Blas, Edwin. (1989). Hábitos de estudio de los estudiantes del primer nivel de Estudios Generales 1988-II. Un estudio exploratorio (copia mimeografiada). Lima: Universidad de Lima.

Salas Blas, Edwin. (1992). Hábitos de estudio en ingresantes a la Universidad de Lima. Un estudio longitudinal de 1988-II a 1991-I. Segundo Encuentro de Psicólogos y Orientadores en Educación Superior.

Salas Blas, Edwin. (1994). Perfil de personalidad de alumnos ingresantes a la Universidad de Lima. Tercer Encuentro de Psicólogos y Orientadores en Educación Superior (documento no publicado.

Salas Blas, Edwin. (1995). Perfil de personalidad de estudiantes que acuden al servicio psicopedagógico en busca de orientación vocacional. Primer Congreso Nacional de Psicología Educativa de Arequipa (documento no publicado).
Salkind, Neil J. (1997). Métodos de investigación. 3a. edición. México: Editorial Prentice Hall Interamericana S.A.

Tapia Mendieta, Violeta. (1999). Efectos de un programa de estrategias cognitivas y metacognitivas para mejorar la comprensión lectora. Revista de la Facultad de Psicología III(4), 9-21. Lima: Universidad Nacional Mayor de San Mar$\cos$.

Universidad de Lima. (1994). Segundo Encuentro de Psicólogos y Orientadores en Educación Superior.

Vera Pérez, Enrique \& Winder Calmet, Erika. (1995). Hábitos de estudio en alumnos desoriantados vocacionalmente. Primer Congreso de Psicología Educativa de Arequipa (documento no publicado), Universidad de Lima.

Vera Pérez, Enrique \& Saenz, Elena. (1997). Capacidad intelectual en alumnos con bajo rendimiento en la asignatura de matemáticas (documento no publicado), Universidad de Lima.

Wechsler, D. (1955). Manual for the Wechsler Adult Intelligence Scale. New York: The Psychological Corporation. 